\title{
Intermittent Heating and Cooling Load Calculation Method comparing with ISO 13790
}

\author{
Sang Hoon Lee \\ College of Architecture, Georgia Institute of Technology, Atlanta, GA, US
}

\begin{abstract}
The intermittent heating and cooling energy need calculation of the ISO 13790 monthly method was examined. The current ISO 13790 method applies a reduction factor to the continuous heating and cooling need calculation result to derive the intermittent heating and cooling for each month. This paper proposes a method for the intermittent energy need calculation based on the internal mean temperature calculation. The internal temperature calculation procedure was introduced considering the heat-balance taking into account of heat gain, heat loss, and thermal inertia for reduced heating and cooling period. Then, the calculated internal mean temperature was used for the intermittent heating and cooling energy need calculation. The calculation results from the proposed method were compared to the current ISO 13790 method and validated with a dynamic simulation using EnergyPlus. The study indicates that the intermittent heating and cooling energy need calculation method using the proposed model improves transparency of the current ISO 13790 method and draws more rational outcomes in the monthly heating and cooling energy need calculation.
\end{abstract}

\section{Keywords : Normative Energy Model, Building Energy Performance, Thermal Energy Need}

\section{INTRODUCTION}

The building sector consumes more than $40 \%$ of the world's primary energy, and this accounts for $24 \%$ of world CO2 emissions (United Nations Environment Programme, 2007). To achieve buildings to be energy efficient, systematic evaluation of energy performance of each building is necessary. This has brought development of energy performance assessment standards which deserve more attention to find out the desirable characteristics of standardized performance indicators. European Committee for Standardization (CEN) and International Organization for Standardization (ISO) have developed the building energy performance assessment standards, which provides the calculation recipe based on the normative calculation method. This approach forms a basis to assess building energy performance by a transparent method (Hogeling \& Dijk, 2008). The development of a simple method to calculate the energy needs for heating and cooling is integrated in the international standard ISO 13790 for

Corresponding Author: Sang Hoon Lee, Research Scientist College of Architecture, Georgia Institute of Technology 2454 th Street. NW, Atlanta Georgia, USA

Tel :+1 4042421208 e-mail : sanghoon.lee@gatech.edu

This is an Open Access article distributed under the terms of the Creative Commons Attribution Non-Commercial License (http://creativecommons. org/licenses/by-nc/3.0/) which permits unrestricted non-commercial use, distribution, and reproduction in any medium, provided the original work is properly cited. the calculation of the energy use for space heating and cooling for residential and non-residential buildings (Dijk, Spiekman, \& Wilde, 2005). ISO 13790 introduces three methods to calculate energy needs; monthly quasi-steady-state calculation method, simple hourly dynamic calculation method, and calculation procedures for detailed simulation method.

Among the three methods, this paper focuses on the monthly quasi-steady-state method which the calculation is based on heat loss by transmission and ventilation, heat grain from solar and internal sources, and utilization factor considering of dynamic parameters. The calculation follows the pre-defined procedure allowing a special regard to the normative reference of heat gains, loss, occupancy, controls, and system efficiencies. This development of the quasi-steady-state method is on the basis of the condition of continuous heating and cooling with a set-point temperature for the whole the calculation period. The standard also introduces the intermittent heating and cooling calculation method with a reduced set-point temperature or switch-off (ISO, 2008). However, this intermittent heating and cooling need calculation method is not comprehensive to cover the internal temperature from dynamic effects from heat gain, loss, and thermal mass. Thus, the author proposes a method to calculate the internal mean temperature which can be applied to the intermittent heating and cooling calculation method.

\section{QUASI-STEADY STATE MONTHLY METHOD IN ISO 13790}

Energy need for heating and cooling is defined as heat to be added to, or extracted from, a conditioned space to maintain the 
intended temperature conditions during a given time in ISO 13790. The purpose of calculating energy need is to assess the energy performance of the building design including envelope, material, ventilation, internal energy use, comfort requirements and controls. In ISO 13790 monthly quasi-steady state method, the energy need for space heating and cooling is introduced as according to equation (1) and (2).

For heating need:

$Q_{H}(t)=\left[\begin{array}{c}\left(H_{t r}+H_{v e}\right)\left(\theta_{i, s e t, h}-\theta_{e}\right) \\ -\eta_{H, g n}\left(Q_{\text {sol }}+Q_{\text {int }}\right)\end{array}\right] t$

For cooling need:

$Q_{C}(t)=\left[\begin{array}{c}\left(Q_{\text {sol }}+Q_{\text {int }}\right)-\eta_{C, l s}\left(H_{t r}+H_{v e}\right) \\ \left(\theta_{i, s e t, c}-\theta_{e}\right)\end{array}\right] t$

Where:

$$
\mathrm{Q}_{\mathrm{H}}(\mathrm{t}) \text { and } \mathrm{Q}_{\mathrm{C}}(\mathrm{t})
$$

$\mathrm{H}_{\mathrm{tr}}$ and $\mathrm{H}_{\mathrm{ve}}$

$\theta_{\mathrm{i}, \mathrm{set}, \mathrm{h}}$ and $\theta_{\mathrm{i}, \mathrm{set}, \mathrm{c}}$

$\theta_{\mathrm{e}}$

$\mathrm{Q}_{\text {sol }}$ and $\mathrm{Q}_{\text {int }}$

$\eta_{H, g n}$ and $\eta_{\mathrm{C}, \mathrm{ls}}$

$t$

Utilization factors for heating and cooling are determined on the basis of the reference numerical parameter $\alpha_{0} \mathrm{H}, \mathrm{C}$ with a suggested value of 1 for both heating and cooling, and the reference time constant $\tau_{0} \mathrm{H}, \mathrm{C}$, with a suggested value of 15 hours for heating and cooling by ISO 13790. The reference values and utilization factors can be optimized from iterative investigation to improve the results of the monthly heating and cooling need calculation results (Jokisalo \& Kurnitski, 2007; Kokogiannakis, Strachan, \& Clarke, 2008).

ISO 13790 introduces internal set-point temperature considerations for the continuous and quasi-continuous heating and cooling energy need calculation. For the quasi-continuous heating and/or cooling, intermittent heating and/or cooling shall be considered as continuous heating and/or cooling with adjusted set-point temperature if mode A or mode B applies as specified in Table 1.
Table 1. Two Modes of Set-point Temperature Calculation in ISO 13790

\begin{tabular}{|c|c|c|}
\hline Mode & Determination & $\begin{array}{c}\text { Set-point temperature } \\
\text { for calculation }\end{array}$ \\
\hline A & $\begin{array}{c}\text { - The set-point temperature variations } \\
\text { between normal heating or cooling } \\
\text { and reduced heating or cooling periods } \\
\text { are less than 3K and/or } \\
\text { - The time constant of the building is } \\
\text { less than 0.2 the duration of the } \\
\text { shortest reduced heating period (for } \\
\text { heating) or cooling period (for } \\
\text { cooling). }\end{array}$ & $\begin{array}{c}\text { Time average of the } \\
\text { set-point } \\
\text { temperatures. }\end{array}$ \\
\hline B & $\begin{array}{c}\text { The time constant of the building is } \\
\text { greater than three times the duration of } \\
\text { the longest reduced heating (cooling) } \\
\text { period. }\end{array}$ & $\begin{array}{c}\text { Set-point temperature } \\
\text { for the normal heating } \\
\text { (cooling) mode. }\end{array}$ \\
\hline
\end{tabular}

In the case of intermittent heating and/or cooling which does not fulfill the conditions in the two modes above, a dimensionless reduction factor is used to calculate the energy needs in the current ISO 13790, based on the fraction of the number of building operation hours and days, empirical correlation factor, time constant, and heat-balance ratio. Equation (3) and (4) are the intermittent energy need calculation using a reduction factor defined by ISO 13790.

$$
\begin{aligned}
& Q_{H, \text { nd,intr }}=a_{H, \text { red }} \cdot Q_{H, \text { nd,cont }} \\
& Q_{C, \text { nd,intr }}=a_{C, \text { red }} \cdot Q_{C, n d, \text { cont }}
\end{aligned}
$$

Where:
$Q_{H, n d, i n t r}$ and

$Q_{C, n d, i n t r}$

$Q_{H, n d, \text { cont }}$ and

$Q_{C, n d, \text { cont }}$

$a_{H, \text { red }}$ and $a_{C, \text { red }}$ the energy need for intermittent heating and cooling respectively;

the energy needs for continuous heating and cooling respectively;

the dimensionless reduction factors for intermittent heating and cooling respectively.

The dimensionless reduction factors for intermittent heating and cooling are calculated as equation (5) and (6).

$$
\begin{aligned}
& a_{H, \text { red }}=1-b_{H, \text { red }}\left(\frac{\tau_{H, 0}}{\tau}\right) \gamma_{H}\left(1-f_{H, h r}\right) \\
& a_{C, \text { red }}=1-b_{C, \text { red }}\left(\frac{\tau_{C, 0}}{\tau}\right) \gamma_{C}\left(1-f_{C, \text { day }}\right)
\end{aligned}
$$

Where: 


$\begin{array}{ll}f_{H, h r} & \begin{array}{l}\text { the fraction of the number of hours in } \\ \text { the week with a normal heating set- } \\ \text { point; } \\ \text { the faction of the number of days in } \\ \text { the week with normal cooling set- } \\ \text { point; } \\ \text { an empirical correlation factors; the } \\ \text { value is } 3 \text {; } \\ \text { the time constant of the building zone in } \\ \text { (hour); } \\ \text { the reference time constants for heating } \\ b_{H, \text { red }} \text { and } b_{C, \text { red }} \\ \tau \\ \tau_{H, 0} \text { and } \tau_{C, 0} \\ \gamma_{H} \text { and } \gamma_{C}\end{array} \quad \begin{array}{l}\text { the heating; } \\ \text { cooling respectively. }\end{array}\end{array}$

The building thermal inertia impact is different for heating and cooling mode due to the diurnal pattern of the external temperature and solar radiation. Generally, this diurnal effect results in temperature setback and switch-off during night time is less effective for cooling mode than heating. This leads to differences between the two formulations of $\mathrm{f}_{\mathrm{H}, \mathrm{hr}}$ and $\mathrm{f}_{\mathrm{C} \text {,day }}$ for the reduction factor calculation (Corrado, Mechri, \& Fabrizio, 2007).

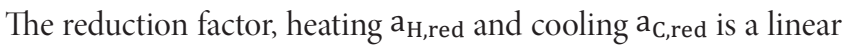
function of the heat balance ratio with the minimum value of $\mathrm{f}_{\mathrm{H}, \mathrm{hr}}$ for heating and $\mathrm{f}_{\mathrm{C}, \text { day }}$ for cooling and the maximum value of one. The reduction factor is multiplied to the total continuous heating and cooling needs respectively to calculate the intermittent heating and cooling energy needs.

Although, ISO 13790 advocates this method, the standard made a remark that an alternative method may be decided to consider the effect of intermittency. As an alternative method, the paper proposes a model to derive the internal mean temperature which can be input in equation (1) and (2) for the intermittent heating and cooling energy need calculation method. The intention of a new model development is to provide a more transparent method to ISO 13790, which follows the fundamental thermodynamics law.

\section{PROPOSED INTERNAL TEMPERATURE CALCULATION METHOD}

\section{(1) Calculation Model}

The internal set-point temperature plays a role in the transmission and ventilation heat transfer calculation. For the continuous heating and cooling, a fixed set-point temperature is used for a given calculation period with a consideration of the utilization factor depending on the thermal inertia and the ratio of the heat gains to the heat losses. However, for the intermittent heating and cooling condition, the internal temperature changes depending on the condition of external temperature, heat transfer, heat gain, and thermal inertia during the reduced set-point temperature or switchoff period during unoccupied. For this reduced heating and cooling period, the internal building free-floating temperature needs to be calculated to adjust the internal set-point temperature for the calculation of intermittent heating and cooling energy needs. The bottom-line energy need calculation procedure adheres to the ISO
13790 method. The core is that the internal mean temperature replaces the fixed set-point temperature for the intermittent heating and cooling need calculation.

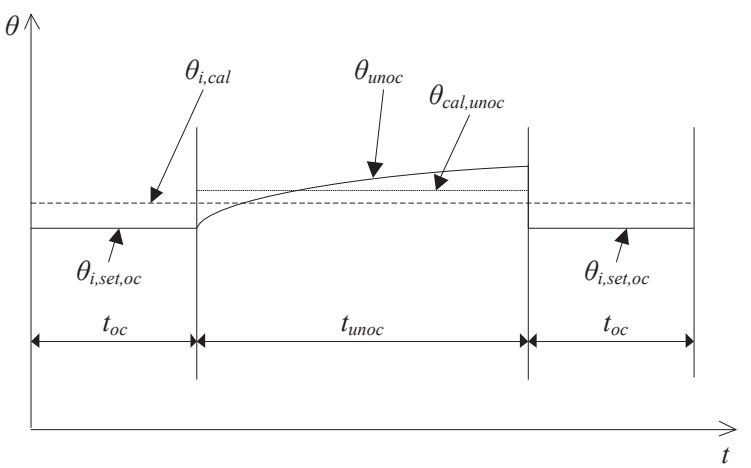

Figure 1. Illustration of the internal temperature for the intermittent cooling

$\begin{array}{ll}\theta_{i, \text { set }, \text { oc }} & \begin{array}{l}\text { internal set-point temperature for the } \\ \text { occupied period; } \\ \text { internal free floating temperature for the } \\ \theta_{\text {unoc }}\end{array} \\ \theta_{\text {cal,unoc }} & \begin{array}{l}\text { unoccupied period; } \\ \text { calculated set-point temperature for the } \\ \text { unoccupied period; } \\ \theta_{i, \text { cal }}\end{array} \\ t_{\text {oc }} & \begin{array}{l}\text { calculated set-point temperature for the entire } \\ \text { calculation period; } \\ \text { duration of occupied period; }\end{array} \\ t_{\text {unoc }} & \text { duration of unoccupied period. }\end{array}$

The proposed calculation model calculates a free floating internal temperature when the heating or cooling system load is reduced or off during the unoccupied period. It is assumed that the system delivers sufficient power to enable to meet the heating and cooling needs. Taking a typical cooling temperature control as an example, Figure 1 illustrates a room temperature during normal system cooling operation for the occupied time and switch-off period for the unoccupied time.

The free floating temperature curve for the unoccupied period is determined by exterior temperature, heat gain/loss, and thermal inertia of building envelope. In this approach, $\theta_{\text {cal,unocc is calculated }}$ as the average room temperature during the unoccupied period, in which room temperature free floats starting from $\theta_{\text {int,set,occ. While }}$ the unoccupied internal temperature $\theta_{\text {unocc }}$ is derived from the heat balance, which is further explained in equation (7) and (8). Equation (7) shows the heat balance equation of the unoccupied period.

$M \frac{d \theta_{\text {unoc }}(t)}{d t}=H_{\text {unoc }} \cdot\left(\theta_{e, \text { unoc }}-\theta_{\text {unoc }}(t)\right)+Q_{\text {gn, unoc }}$ 

M
$t$
$H_{\text {unoc }}$
$\theta_{e, \text { unoc }}$
$\theta_{\text {unoc }}(t)$
$Q_{\text {gn,unoc }}$

Thus, $\theta_{\text {unocc }}(\mathrm{t})$ is derived from:

$$
\begin{aligned}
& \theta_{\text {unoc }}(t)=\left(\theta_{i, \text { set }}-\theta_{e, \text { unoc }}-\frac{Q_{\text {gn }, \text { unoc }}}{H_{\text {unoc }}}\right) e^{-\frac{H_{\text {unoc }}}{M} t} \\
& +\theta_{e, \text { unoc }}+\frac{Q_{\text {gn }, \text { unoc }}}{H_{\text {unoc }}}
\end{aligned}
$$

Based on equation (8), $\theta_{\text {cal,unocc }}(t)$ is calculated as:

$$
\begin{array}{r}
\theta_{\text {cal }, \text { unoc }}(t)=\frac{M}{t \cdot H_{\text {unoc }}}\left(\theta_{i, s e t}-\theta_{e, \text { unoc }}-\frac{Q_{\text {gn,unoc }}}{H_{\text {unoc }}}\right)(1 \\
-e^{\left.-\frac{H_{\text {unoc }}}{M} \cdot t\right)}+\theta_{e, \text { unoc }}+\frac{Q_{\text {gn, unoc }}}{H_{\text {unoc }}}
\end{array}
$$

Therefore, the adjusted internal set-point temperature $\theta_{\text {cal,int,set }}$ is calculated as:

For heating mode:

$$
\theta_{i, c a l, \mathrm{~h}}=\frac{\theta_{\mathrm{i}, \text { set }, \mathrm{h}} t_{o c}+\theta_{\text {cal }, \text { unoc }} t_{\text {unoc }}}{t_{o c}+t_{\text {unoc }}}
$$

For cooling mode:

$$
\theta_{i, c a l, \mathrm{c}}=\frac{\theta_{\mathrm{i}, \mathrm{set}, \mathrm{c}} t_{o c}+\theta_{\text {cal }, \text { unoc }} t_{\text {unoc }}}{t_{o c}+t_{\text {unoc }}}
$$

$\theta_{\mathrm{i}, \text { cal }}$ is the time average temperature for the calculation period considering night time and weekend free-floating temperature.

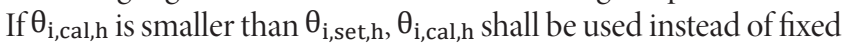
set-point temperature $\theta_{i, s e t, h}$ in equation (1) for the intermittent heating need calculation. If $\theta_{i, c a l, c}$ is greater than $\theta_{i, s e t, c}, \theta_{i, c a l, c}$ shall be used instead of fixed set-point temperature $\theta_{i, \text { set,c }}$ in equation (2) for the intermittent cooling need calculation.

If $\theta_{\mathrm{i}, \mathrm{cal}, \mathrm{hH}}$ is greater than $\theta_{\mathrm{i}, \mathrm{set}} \mathrm{h}$, the intermittent heating need calculation follows equation (3). If $\theta_{i, c a l, c}$ is smaller than $\theta_{i, s e t, c}$ , the intermittent cooling need calculation follows equation (4). For heating mode, when the calculated mean temperature for the calculation period is greater than the heating set-point temperature typically during hot months, the calculated internal temperature during unoccupied period is higher than the heating set-point temperature. For cooling mode, when the calculated mean temperature is smaller than the cooling set-point temperature normally during cold months, the calculated internal temperature during unoccupied period smaller than the cooling set-point temperature. In this case, heating or cooling system is switch-off reduction factor shall be applied considering the operation faction, heat balance ratio, and time constant as specified in equation (5) for heating and (6) for cooling.

\section{(2) Case Study}

To test the proposed intermittent energy need calculation using the adjusted internal set-point temperature, the study uses an exemplary building as a case study (shown as Figure 2).



Figure 2. Example building used for the energy performance assessment study

The exemplary building is chosen under the strategy to avoid increasing the complexity of the calculations with regards to the simplified methods. Also, this strategy enables the validation with a dynamic simulation program realistic minimizing the complexity of dynamic interactions between the thermal zones (Kokogiannakis et al., 2008). The input parameters for the case study are specified in Table 2.

Table 2. Parameters of the exemplary building

\begin{tabular}{cc}
\hline Parameter & Value \\
\hline Location & Atlanta, GA, US \\
Total conditioned floor area $\left[\mathrm{m}^{2}\right]$ & 336 \\
Ventilated volume $\left[\mathrm{m}^{3}\right]$ & 840 \\
Roof area $\left[\mathrm{m}^{2}\right]$ & 112 \\
Ground floor area $\left[\mathrm{m}^{2}\right]$ & 112 \\
Number of Floors & 3 (ground, mid, top) \\
Internal heat gain: occupied $\left[\mathrm{W} / \mathrm{m}^{2}\right]$ & 22 \\
Internal heat gain: unoccupied $\left[\mathrm{W} / \mathrm{m}^{2}\right]$ & 2.2 \\
Envelop area: glazing / opaque $\left[\mathrm{m}^{2}\right]$ & \\
South & $31.5 / 73.5$ \\
East & $18 / 42$ \\
North & $31.5 / 73.5$ \\
West & $18 / 42$ \\
Roof U-value $\left[\mathrm{W} /\left(\mathrm{Km}^{2}\right)\right]$ & 0.25 \\
Opaque wall U-value $\left[\mathrm{W} /\left(\mathrm{Km}{ }^{2}\right)\right]$ & 0.35 \\
Glazing U-value $\left[\mathrm{W} /\left(\mathrm{Km}^{2}\right)\right]$ & 3.16 \\
Glazing Solar Transmittance & $0.6(\mathrm{SHGC:} 0.69)$ \\
Ventilation constant throughout the year $[$ ac $/ \mathrm{h}]$ & 0.7 \\
Cooling set-point: occupied $\left[{ }^{\circ} \mathrm{C}\right]$ & 24 \\
Cooling set-point: unoccupied $\left[{ }^{\circ} \mathrm{C}\right]$ & 29 \\
Heating set-point: occupied $\left[{ }^{\circ} \mathrm{C}\right]$ & 21 \\
Heating set-point: unoccupied $\left[{ }^{\circ} \mathrm{C}\right]$ & 16 \\
Heat capacity $\left[\mathrm{J} /\left(\mathrm{Km}^{2}\right)\right]$ & $165,000$ (Medium $)$ \\
Building occupied hours $[$ Hours] & $10(8 \mathrm{am}-6 \mathrm{pm}$ with Mon-Fri) \\
\hline &
\end{tabular}


The exemplary building is an office type three-story building located in Atlanta, Georgia in United States. The conditioned gross floor area is $336 \mathrm{~m}^{2}$ and ventilated volume is $840 \mathrm{~m}^{3}$. Double glazing and medium heat capacity wall of $165 \mathrm{KJ} / \mathrm{m}^{2} \mathrm{~K}$ as categorized in ISO 13790 with an overall solar absorption coefficient of 0.9 was used for building envelope. The internal set-point temperature during occupied periods for heating is $21^{\circ} \mathrm{C}$ and cooling $24^{\circ} \mathrm{C}$, and the setback temperature during unoccupied periods is $16^{\circ} \mathrm{C}$ and $29^{\circ} \mathrm{C}$ respectively. The operation of the building starts at $8 \mathrm{am}$ and ends $6 \mathrm{pm}$ for 10 hours. The ventilation rate is $0.7 \mathrm{ACH}$ throughout the year and corresponds to 163 liter/s. Total internal heat gain during occupied period is $22 \mathrm{~W} / \mathrm{m}^{2}, 10 \%$ of which $\left(2.2 \mathrm{~W} / \mathrm{m}^{2}\right)$ is used for unoccupied.

\section{(3) Internal Temperature Calculation}

The internal temperature for unoccupied periods was calculated from equation (7) and (8). The average ambient temperature, heat transfer coefficient, internal and solar heat gain during the unoccupied period for 14 hours from $6 \mathrm{pm}$ to $8 \mathrm{am}$ for a weekday nighttime were required for the calculation. For the weekend unoccupied period, the calculation input shall use separate parameters of Friday night, Saturday daytime and nighttime, and Sunday daytime and nighttime. Table 3 shows the monthly average exterior temperatures for daytime and nighttime required for the calculation.

Table 3. Monthly average exterior temperature for daytime and nighttime for Atlanta

\begin{tabular}{cccc}
\hline & $\theta_{e}$ & $\theta_{e, \text { day }}$ & $\theta_{e, \text { night }}$ \\
\hline Jan & 4.0 & 5.2 & 3.1 \\
Feb & 7.9 & 9.5 & 6.9 \\
Mar & 13.8 & 15.3 & 12.6 \\
Apr & 17.2 & 20.4 & 14.9 \\
May & 20.8 & 23.9 & 18.6 \\
Jun & 24.8 & 28.1 & 22.4 \\
Jul & 26.1 & 28.8 & 24.1 \\
Aug & 26.5 & 29.0 & 24.8 \\
Sep & 22.5 & 24.9 & 20.8 \\
Oct & 16.0 & 18.6 & 14.1 \\
Nov & 11.9 & 14.3 & 10.3 \\
Dec & 7.7 & 9.4 & 6.5 \\
\hline
\end{tabular}

Figure 3 and Figure 4 for heating mode and Figure 5 and Figure 6 for cooling mode illustrate the calculated internal temperature $\theta_{\text {unocc }}(t)$ for each month during the unoccupied period for weekday and weekend.

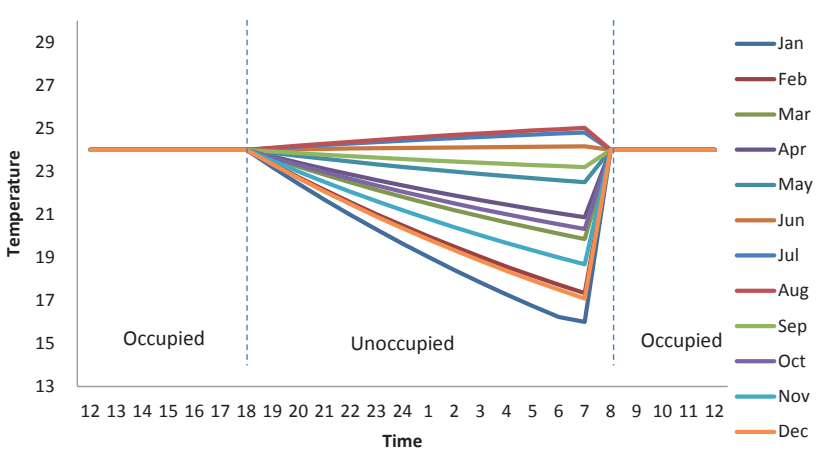

Figure 3. Calculated internal temperature by hour during weekday unoccupied periods for heating mode



Figure 4. Calculated internal temperature by hour during weekend unoccupied periods for heating mode

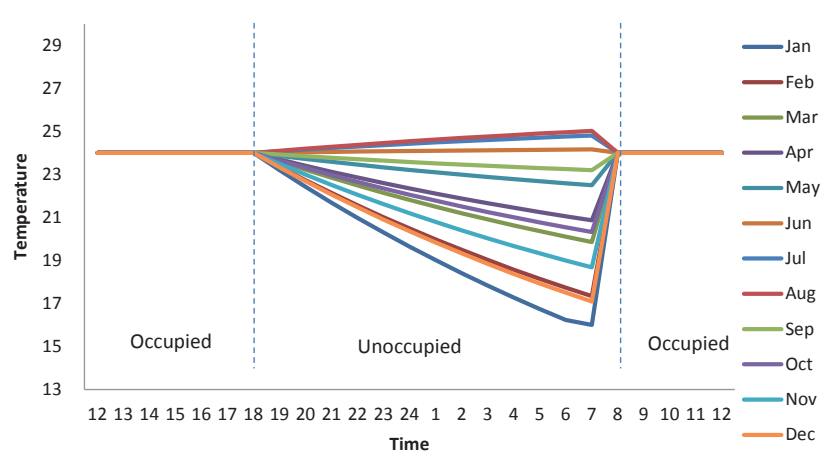

Figure 5. Calculated internal temperature by hour during weekday unoccupied periods for cooling mode

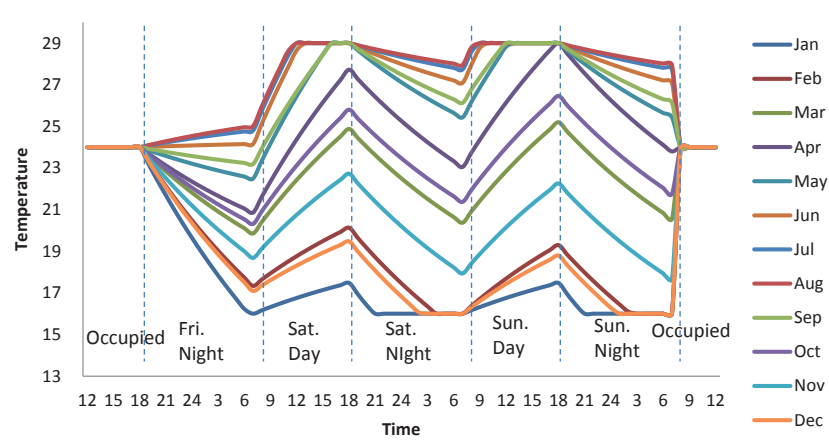

Figure 6. Calculated internal temperature by hour during weekend unoccupied periods for cooling mode 
The average unoccupied internal temperatures for weekday nighttime and weekend are calculated using the equation (8), and the average internal temperature for the calculation period is calculated using the equation (9). Then, the temperature $\theta_{\text {cal,int,heat }}$

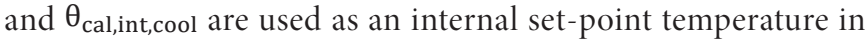
equation (1) and (2) for the intermittent heating and cooling need calculation for applicable months.

Table 4 shows the monthly calculated temperatures for heating mode and Table 5 for cooling mode. The comparison between the calculated mean internal temperature and the set-point temperature determine which method shall be chosen.

Table 4. Calculated internal temperature for monthly heating energy need calculation input

\begin{tabular}{c|ccc}
\hline Month & $\theta_{\text {int,set, } \mathrm{H}}$ & $\theta_{\text {cal,int, } \mathrm{H}}$ & Method \\
\hline \hline Jan & 21.0 & 18.3 & Proposed Model \\
Feb & 21.0 & 18.8 & Proposed Model \\
Mar & 21.0 & 20.6 & Proposed Model \\
Apr & 21.0 & 21.7 & ISO 13790 \\
May & 21.0 & 22.6 & ISO 13790 \\
Jun & 21.0 & 23.3 & ISO 13790 \\
Jul & 21.0 & 23.5 & ISO 13790 \\
Aug & 21.0 & 23.6 & ISO 13790 \\
Sep & 21.0 & 22.9 & ISO 13790 \\
Oct & 21.0 & 21.0 & ISO 13790 \\
Nov & 21.0 & 19.6 & Proposed Model \\
Dec & 21.0 & 18.6 & Proposed Model \\
\hline
\end{tabular}

Table 5. Calculated internal temperature for monthly cooling energy need calculation input

\begin{tabular}{c|ccc}
\hline Month & $\theta_{\text {int,set, }}$ & $\theta_{\text {cal,int, }}$ & Method \\
\hline \hline Jan & 24.0 & 19.99 & ISO 13790 \\
Feb & 24.0 & 20.70 & ISO 13790 \\
Mar & 24.0 & 22.68 & ISO 13790 \\
Apr & 24.0 & 23.75 & ISO 13790 \\
May & 24.0 & 24.58 & Proposed Model \\
Jun & 24.0 & 25.22 & Proposed Model \\
Jul & 24.0 & 25.45 & Proposed Model \\
Aug & 24.0 & 25.52 & Proposed Model \\
Sep & 24.0 & 24.83 & Proposed Model \\
Oct & 24.0 & 23.08 & ISO 13790 \\
Nov & 24.0 & 21.72 & ISO 13790 \\
Dec & 24.0 & 20.54 & ISO 13790 \\
\hline
\end{tabular}

(4) Intermittent Heating and Cooling Calculation Result Analysis

Above all, time constant and duration was checked for the application of the intermittent method as specified in Table 1. If the time constant of the building is greater than three times the duration of the longest reduced heating and cooling period, the set-point temperature for the intermittent energy need calculation is same as the temperature for the continuous energy need calculation. The longest reduced heating and cooling periods on weekend are 62 hours and the time constant is 24.4 hours. This leads to require the intermittent heating and cooling need calculation.

Firstly, the monthly continuous heating and cooling energy needs were calculated with internal set-point temperature $24^{\circ} \mathrm{C}$ for cooling and $21^{\circ} \mathrm{C}$ for heating throughout the year. The exemplary building with the Atlanta climate zone has a dominant cooling need than heating. The yearly total energy need per conditioned area is $33 \mathrm{kWh} / \mathrm{m} 2$ for continuous heating and $99 \mathrm{kWh} / \mathrm{m} 2$ for continuous cooling. Secondly, ISO 13790 method for the whole the year each month was applied using equation (3), (4), (5), and (6). Thirdly, the intermittent heating and cooling needs were calculated with the proposed model. The proposed model checked the calculated monthly mean internal temperature in for heating and cooling mode, and the selected method was applied in each month as specified in Table 4 for heating and Table 5 for cooling.
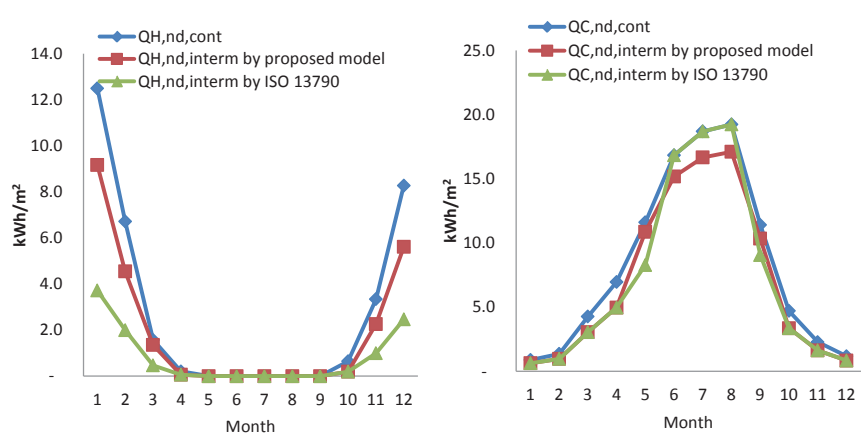

Figure 7. Monthly heating (left) and cooling (right) need calculation result $\left(\mathrm{kWh} / \mathrm{m}^{2} / \mathrm{month}\right)$

Figure 7 illustrates the monthly heating and cooling energy needs calculation results for three cases. For heating, the intermittent heating need calculation result by the proposed model and the ISO 13790 method shows respectively $71 \%$ $\left(23 \mathrm{kWh} / \mathrm{m}^{2}\right)$ and $31 \%\left(10 \mathrm{kWh} / \mathrm{m}^{2}\right)$ compared to the continuous heating. For cooling, the proposed intermittent cooling need calculation result is $86 \%\left(86 \mathrm{kWh} / \mathrm{m}^{2}\right)$, and the ISO 13790 method is $88 \%\left(88 \mathrm{kWh} / \mathrm{m}^{2}\right)$.

Heat transfer by transmission and ventilation causes heat gain for hot months. The month of June, July, and August shows heat gains from heat transfer for this case study. When the months heat transfer cause heat gain, the parameter for the heat balance ratio $\gamma_{\mathrm{H}}$ for heating and $\gamma_{\mathrm{C}}$ for cooling becomes negative with the current ISO 13790 method. This forces the reduction factor $\mathrm{a}_{\mathrm{H}, \mathrm{red}}$

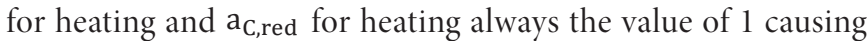
no reduction for intermittent. In cooling (right) calculation result depicts that the intermittent cooling need is same as continuous cooling need for the month of June, July and August.

Also, the case study conducted energy need calculation to see the effect of heat capacity. Envelope structures varying from very light to very heavy weights according to ISO 13790 heat capacity classes as specified in Table 6 were used for the calculation. Continuous, intermittent with a proposed model, and ISO 13790 intermittent method calculations were conducted. 
Table 6. Different heat capacity class

\begin{tabular}{ccc}
\hline Class & Heat capacity $\left(\mathrm{J} / \mathrm{Km}^{2}\right)$ & Time constant (hour) \\
\hline Very Light & 80,000 & 11.82 \\
Light & 110,000 & 16.26 \\
Medium & 165,000 & 24.38 \\
Heavy & 260,000 & 38.42 \\
Very heavy & 370,000 & 54.68 \\
\hline
\end{tabular}

Figure 8 shows the annual heating and cooling energy needs. For the calculation, all other input parameters remain as described in Table 2.
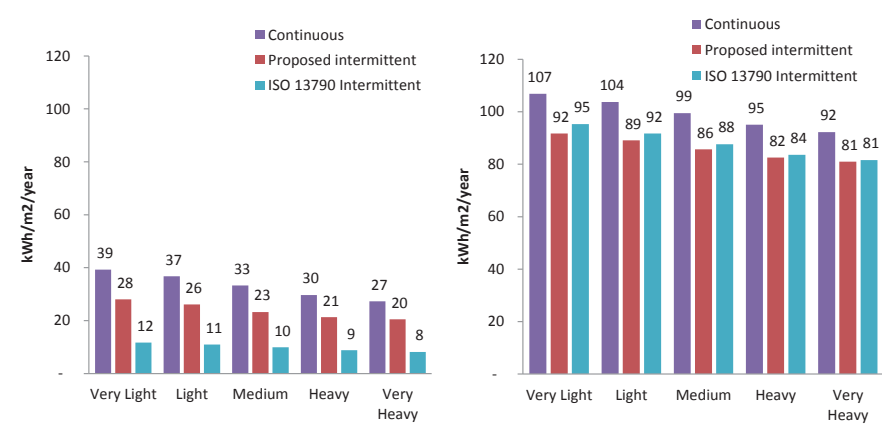

Figure 8. Effect of heat capacity on heating (left) and cooling (right) needs $\left(\mathrm{kWh} / \mathrm{m}^{2} /\right.$ year)

The EnergyPlus simulation was conducted to validate the proposed intermittent energy need calculation study. All the inputs used for the monthly calculation were best represented to the simulation. The cooling temperature schedule was set to $24^{\circ} \mathrm{C}$ for the duration of occupied and $29^{\circ} \mathrm{C}$ for the unoccupied, and the heating is set to $21{ }^{\circ} \mathrm{C}$ for occupied and $16^{\circ} \mathrm{C}$ for unoccupied. The cooling and heating load simulation outcomes were compared to the intermittent energy need calculation with the proposed model and ISO 13790. Figure 9 presents the monthly comparison for heating and cooling need for the example case located in Atlanta.
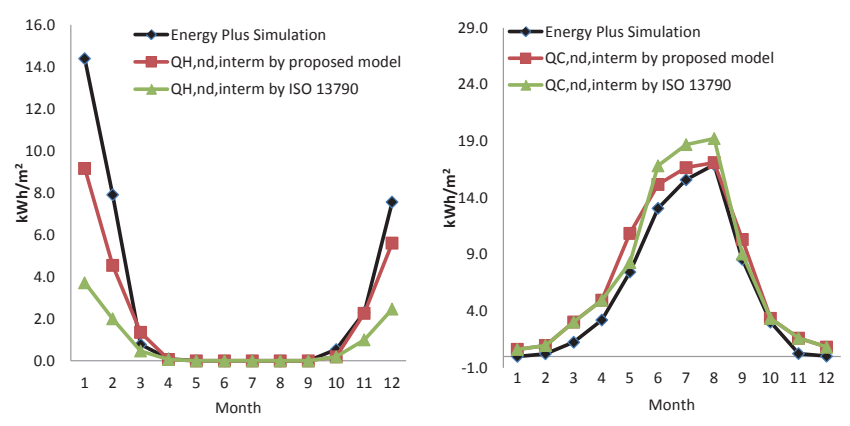

Figure 9. Intermittent heating need monthly calculation result comparison with simulation $\left(\mathrm{kWh} / \mathrm{m}^{2} /\right.$ month)

Atlanta belongs to the ASHRAE climate zone $3 \mathrm{~A}$ which represents a warm weather condition. The study further investigated to test the proposed model applying different climate locations, still all other inputs remain the same. Chosen cities are Miami, Florida representing a hot and humid region and Helena, Montana cold and dry. Each belongs to the ASHRAE climate zone $1 \mathrm{~A}$ and $6 \mathrm{~B}$. Figure 10 illustrates the yearly total heating and cooling needs from the proposed model, ISO 13790 method and EnergyPlus simulation for three cities.

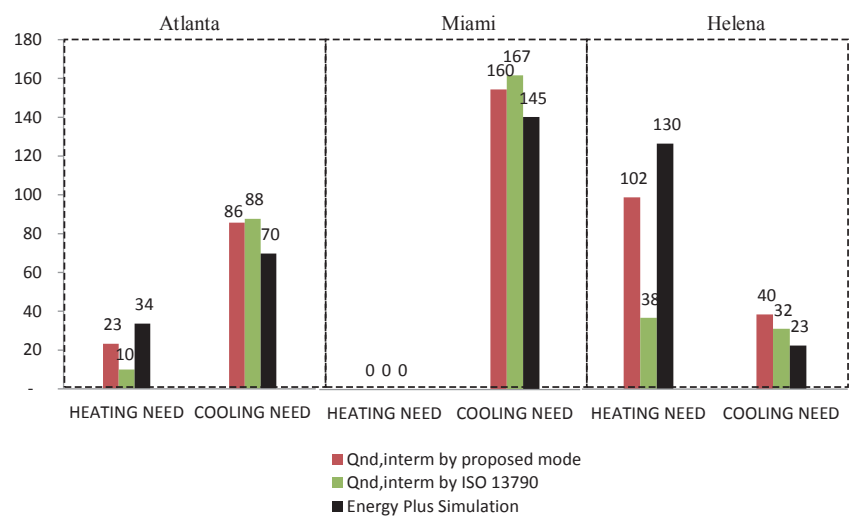

Figure 10. Total thermal energy need result $\left(\mathrm{kWh} / \mathrm{m}^{2} /\right.$ year $)$

The proposed intermittent energy need calculation maintains a more reliable margin of difference than the ISO 13790 method when compared to the simulation result. The heating need from the ISO 13790 method shows a big difference in Atlanta and Helena. However the ISO 13790 method intermittent cooling result is close to the simulation in Helena case, the calculation shall take a special consideration to derive a faultless reduction factor. Although, the proposed model still follows some normative procedure to calculate the monthly internal mean temperature considering occupied setpoint and unoccupied free-floating temperature, this proposed method can be observed more transparent than the ISO 13790 reduction factor method.

\section{CONCLUSIONS}

The normative calculation methods guided by the CEN and ISO standards offer advantages to assess building energy performance in a quick, robust, and transparent way. However, this raises the issue of applicability of the pre-defined equations using the default values and suggested by standards. This paper investigated the intermittent thermal energy need calculation method provided by ISO 13790 standard and proposed a new approach. The approach uses a calculated internal mean temperature considering the occupied set-point and unoccupied free-floating temperature to replace a fixed set-point temperature. Various heating and cooling need analyses were conducted comparing the proposed model and the current ISO 13790 method. Also, the study validated the proposed method with a dynamic simulation using EnergyPlus. An example office building with simplified parameters was used to compare the results from different normative calculation methods and simulations. The proposed intermittent energy needs calculation results maintain a reliable margin of the difference compared to the ISO 13790 method. The applicability of the intermittent calculation method by ISO 13790 using a reduction factor from operational factors and empirical values was found to be questionable. From the study in this paper, the intermittent energy needs calculation using the proposed model improves transparency of the current ISO 13790 method and draws more rational calculation results for monthly heating and cooling energy needs. 


\section{REFERENCES}

Corrado, V., Mechri, H. E., \& Fabrizio, E. (2007). Building energy performance performance assessment thourgh simplified models: application of the ISO 13790 quasi-steady sate method. Paper presented at the IBPSA, Beijing, China.

Dijk, H. v., Spiekman, M., \& Wilde, P. d. (2005). A monthly method for calculating energy performance in the context of European building regulations. Paper presented at the IBPSA, Montreal, Canada.

Hogeling, J., \& Dijk, D. v. (2008). P60 More information on the set of CEN standards for the EPBD.

ISO. (2008). ISO 13790:2008 Energy performance of buildings Calculation of energy use for space heating and cooling.

Jokisalo, J., \& Kurnitski, J. (2007). Performance of EN ISO 13790 utilisation factor heat demand calculation method in a cold climate. Energy and Buildings, 39, 236-247.

Kokogiannakis, G., Strachan, P., \& Clarke, J. (2008). Comparison of the simplified methods of the ISO 13790 standard and detailed modelling programs in a regulatory context Journal of Building Performance Simulation, 1(4), 209-219.

United Nations Environment Programme. (2007). Buildings and Climate Change: Status, Challenges and Opportunities: UNEP/ Earthprint.

(Date of Submission : 2011.10.26) 